\title{
Nonlinear Torsional Vibration Dynamics Behaviors of Rolling Mill's Multi-DOF Main Drive System under Parametric Excitation
}

\author{
Dongying Han, ${ }^{1}$ Peiming Shi, ${ }^{2}$ and Kewei Xia ${ }^{2}$ \\ ${ }^{1}$ College of Vehicles and Energy, Yanshan University, Qinhuangdao 066004, China \\ ${ }^{2}$ College of Electrical Engineering, Yanshan University, Qinhuangdao 066004, China \\ Correspondence should be addressed to Dongying Han; hspace@ysu.edu.cn
}

Received 7 June 2014; Accepted 11 August 2014; Published 24 August 2014

Academic Editor: Zhiwei Gao

Copyright (C) 2014 Dongying Han et al. This is an open access article distributed under the Creative Commons Attribution License, which permits unrestricted use, distribution, and reproduction in any medium, provided the original work is properly cited.

Considering the influence caused by joint angle, nonlinear damping, and nonlinear rigidity, the nonlinear torsional vibration dynamical modeling of the multi-DOF rolling mill's main drive system is established. To analyze the coupled equations by analytic method, the equations are decoupled by transforming them into principal coordinates. The amplitude-frequency characteristic equations are obtained by multiscale method. Furthermore, numerical example based on the 1780 rolling mill's main drive system of some Steel Co. is given to illustrate the effects of the resonance on the response of the system. The relationship between amplitude and frequency varies according to the parameters changes of nonlinear stiffness, nonlinear friction damping, torque disturbance, and joint angle. During the rolling process, the limited joint angles range is obtained and the variation rules of the joint angle caused by the nonlinear damping, nonlinear stiffness, and the disturbance torque are gained. The results present that the rolling mill can work more stably with the joint angle at a range from $2^{\circ}$ to $8^{\circ}$ by controlling the value of parameters. The research results provide a theoretical basis and reference for analyzing torsional vibration of rolling mill's transmission system caused by joint angle.

\section{Introduction}

In recent years, there has been a spurt of interest in the area of modeling and control of complex dynamic systems due to its challenging features and many applications [13]. Rolling mill's main drive system is a complex dynamic system, which is the key and core equipment in iron and steel industry. As the continuous increasing of the rolling speed and strength, torsional vibration phenomenon in rolling mills becomes very common and complicated. The phenomenon severely affects rolling efficiency and product quality. The torsional vibration of rolling mill's main drive systems has been investigated during the past. The self-excited vibration of hot strip mill was studied, and the variation of the rolling torque caused by rolling speed was obtained [4]. A method for calculating the steady-state response of the selfexcited vibration was presented, and the relationship between the system parameters and the self-excited vibration was analyzed [5]. A universal nonlinear mathematical model for the main drive train of a rolling mill was established [6]. The nonlinear torsional vibration characteristic of the hot strip mill's main drive system was examined, and the dynamic model analysis showed that the torsional vibration presented as the movement of the unstable limit cycle [7]. The existence and uniqueness of the limit cycle of the torsional vibration differential equations on rolling mill's main drive system were discussed, and the self-excited vibration of the statistical properties by simplifying the rolling mill's main drive system to a single degree of freedom system was analyzed [8]. The torsional vibration problem of lathe spindle system with unbalanced workpiece was studied [9]. Torsional vibration analysis about axis of the rolls on the transfer function of variable-speed rolling mill motor with shaft systems was studied [10]. The nonlinear torsional vibration characteristics caused by the strip rolling mill's drive trains with angular and radial backlash were investigated, and the torsional vibration fault feature caused by backlash was analyzed under static and dynamic rolling load [11]. 
However, during rolling process, the variation of the joint angle is inevitable during regulating motor speed and rolling strip because of the structural complexity of rolling mill's main drive system. Therefore, it is necessary to study the torsional vibration caused by the presence of the joint shaft in the main drive system of rolling mill.

The paper proceeds as follows. The dynamical model of rolling mill's multi-DOF main drive system is established in Section 2. In Section 3, the amplitude-frequency characteristic equation is obtained by multiscale method. Simulation results to illustrate the results are presented in Section 4. Conclusions are provided in Section 5.

\section{Dynamical Model of Rolling Mill's Multi-DOF Main Drive System}

Considering the influence of the joint angle, as shown in Figure 1, the main drive system of rolling mill can be regarded as an $n$-DOF system. This rotational system with a joint angle of $\alpha$ is driven at the angular frequency $\omega$ of the motor in the rolling mill. The quantities $\psi_{n-1}$ and $\psi_{n}$ represent the input and the output rotation angles of the joint shaft, respectively, while $\theta_{n-1}$ and $\theta_{n}$ symbolize the input and output rotation angles of the system. The moment of inertia in the rolling mill is expressed as $J_{n}$. The joint shaft has been considered to embody torsional stiffness $k_{n-1}$, nonlinear stiffness $K_{\mathrm{NL}}$, linear damping $c_{n-1}$, and nonlinear damping $C_{\mathrm{NL}}$.

The well-known kinematic relationship between the angular displacements of the input and output shaft converging on the joint angle is determined [12]:

$$
\tan \psi_{i}=\tan \theta_{i} \cdot \cos \alpha, \quad i=1,2,
$$

where the angles of joint shaft of rolling mill are between $2^{\circ}$ and $8^{\circ}$.

Then the relationships between motor and input of the joint shaft and roller and output of the joint shaft are

$$
\begin{gathered}
\frac{d \psi_{n-1}}{d \theta_{n-1}}=\frac{\dot{\psi}_{n-1}}{\dot{\theta}_{n-1}}=\frac{\cos \alpha}{1-\sin ^{2} \alpha \sin ^{2} \theta_{n-1}}=\lambda_{n-1}\left(\theta_{n-1}\right), \\
\frac{d \psi_{n}}{d \theta_{n}}=\frac{\dot{\psi}_{n}}{\dot{\theta}_{n}}=\frac{\cos \alpha}{1-\sin ^{2} \alpha \sin ^{2} \theta_{n}}=\lambda_{n}\left(\theta_{n}\right),
\end{gathered}
$$

where $\dot{\theta}_{n-1}, \dot{\theta}_{n}$ denote the angular velocity of the joint shaft and the roller, respectively. $M_{n-1, n}$ is the elastic restoring torque of joint shaft acting on the roller:

$$
M_{n-1, n}=K \cdot \lambda_{n}\left(\theta_{n}\right)\left(\psi_{n}-\psi_{n-1}\right)=K T\left(\theta_{n}, t\right) .
$$

From (2) and (3), an equation of relative angle can be obtained as follows:

$$
\begin{aligned}
T(\Delta \theta, t)= & \frac{\cos \alpha}{1-\sin ^{2} \alpha \cdot \sin ^{2}(\omega t-\Delta \theta)} \\
& \cdot \tan ^{-1} \frac{\cos \alpha[\tan (\omega t-\Delta \theta)-\tan \omega t]}{1+\cos ^{2} \alpha \cdot \tan (\omega t-\Delta \theta) \cdot \tan \omega t} .
\end{aligned}
$$

Due to the fact that the relative angle $\Delta \theta$ is small enough, we can suppose $\tan \Delta \theta \approx \Delta \theta, \tan \left(\psi_{n}-\psi_{n-1}\right) \approx \psi_{n}-\psi_{n-1}$, and $1-\sin ^{2} \alpha \sin ^{2}(\omega t-\Delta \theta) \approx 1-\sin ^{2} \alpha \sin ^{2} \omega t$.

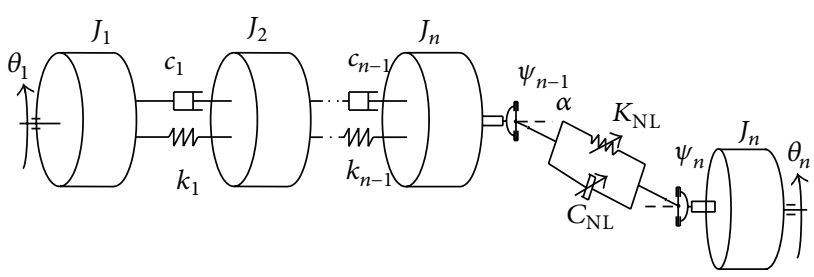

FIGURE 1: Model of multi-DOF main drive system with joint shaft.

Then (4) can be rewritten as

$$
\begin{aligned}
T(\Delta \theta, t)= & \lambda_{1}^{2}(\omega t) \Delta \theta \\
= & {\left[1-\sin ^{2} \alpha \cos 2 \omega t\right.} \\
& +\sin ^{4} \alpha\left(\frac{1}{8}-\frac{1}{2} \cos 2 \omega t+\frac{3}{8} \cos 4 \omega t\right) \\
& \left.+\mathrm{O}\left(\varepsilon^{2}\right)\right] \Delta \theta .
\end{aligned}
$$

For the typical rolling stand modeling shown in Figure 1, its matrix equation of motion is expressed as

$$
\mathbf{J} \ddot{\theta}+\mathbf{C} \dot{\theta}+\mathbf{K} \theta=\mathbf{F},
$$

where $\mathbf{F}$ is the external excitation vector and can be expressed as

$$
\mathbf{F}=\left[\begin{array}{c}
M \cos \omega t \\
0 \\
\vdots \\
-k_{n-1} T_{n-1, n}-K_{\mathrm{NL}}\left(\theta_{n-1}-\theta_{n}\right)^{3}-C_{\mathrm{NL}}\left(\theta_{n-1}-\theta_{n}\right)^{3} \\
-k_{n-1} T_{n, n-1}-K_{\mathrm{NL}}\left(\theta_{n}-\theta_{n-1}\right)^{3}-C_{\mathrm{NL}}\left(\theta_{n}-\theta_{n-1}\right)^{3}
\end{array}\right] .
$$

It is hard to solve the equations because $\mathbf{C}$ and $\mathbf{K}$ are not diagonal matrix. So we decouple (6) into a simple equation in principal coordinates

$$
J_{p i} \ddot{\theta}_{p i}+C_{p i} \dot{\theta}_{p i}+K_{p i} \theta_{p i}=\sum_{j=1}^{n} F_{j} \phi_{j i},
$$

where $\phi$ is the regular modal vector, $F_{p i}=\sum_{j=1}^{n} F_{j} \phi_{j i}, K_{p i}=$ $J_{p i} P_{i}^{2}, J_{p i}=\sum_{j=1}^{n} J_{j} \phi_{j i}^{2}$, and $C_{p i}=2 \zeta_{i} P_{i} J_{p i}$, while $\zeta_{i}$ is the modal ratio of damping.

Substituting $\theta_{n}=\phi_{n i} \theta_{p i}$ into (8), we can obtain

$$
\begin{aligned}
& J_{p i} \ddot{\theta}_{p i}+C_{p i} \dot{\theta}_{p i} \\
& +\left\{K_{p i}+K_{n-1}\left[1-\sin ^{2} \alpha \cos 2 \omega t\right.\right. \\
& \left.+\sin ^{4} \alpha\left(\frac{1}{8}-\frac{1}{2} \cos 2 \omega t+\frac{3}{8} \cos 4 \omega t\right)\right] \\
& \left.\quad \times\left(\varphi_{n, i}-\varphi_{n-1, i}\right)^{2}\right\} \theta_{p i}+K_{\mathrm{NL}}\left(\varphi_{n, i}-\varphi_{n-1, i}\right)^{4} \theta_{p i}^{3} \\
& +C_{\mathrm{NL}}\left(\varphi_{n, i}-\varphi_{n-1, i}\right)^{4} \dot{\theta}_{p i}^{3}=M \varphi_{1, i} \cos \omega t .
\end{aligned}
$$

Thus the equations are decoupled. 


$$
\begin{aligned}
& \text { Suppose } x=\theta_{p i} ;(9) \text { can be expressed as } \\
& \begin{aligned}
\ddot{x}+a_{1} \dot{x}+\omega_{0}^{2} x+a_{2} \\
\times\left[-\sin ^{2} \alpha \cos 2 \omega t\right. \\
\left.\quad+\sin ^{4} \alpha\left(\frac{1}{8}-\frac{1}{2} \cos 2 \omega t+\frac{3}{8} \cos 4 \omega t\right)\right] x \\
+a_{3} x^{3}+a_{4} \dot{x}^{3} \\
=M_{1} \cos \omega t,
\end{aligned}
\end{aligned}
$$

where $a_{1}=C_{p i} / J_{p i}, a_{2}=k_{n-1}\left(\phi_{n, i}-\phi_{n-1, i}\right)^{2} / J_{p i}, a_{3}=$ $K_{\mathrm{NL}}\left(\phi_{n, i}-\phi_{n-1, i}\right)^{4} / J_{p i}, a_{4}=C_{\mathrm{NL}}\left(\phi_{n, i}-\phi_{n-1, i}\right)^{4} / J_{p i}, \omega_{0}=$ $\sqrt{\left(K_{p i}+K_{\mathrm{NL}}\left(\phi_{n, i}-\phi_{n-1, i}\right)^{2}\right) / J_{p i}}$, and $M_{1}=M \phi_{1 i} / J_{p i}$.

\section{Amplitude-Frequency Equation}

A perturbation parameter $\varepsilon$ is embedded to analyze (12) by using the multiple-scales method. So the equation can be expressed as

$$
\begin{aligned}
& \ddot{x}+\omega_{0}^{2} x \\
& =-\varepsilon\left\{a_{1} \dot{x}+a_{2}\right. \\
& \times\left[-\sin ^{2} \alpha \cos 2 \omega t\right. \\
& \left.\quad+\sin ^{4} \alpha\left(\frac{1}{8}-\frac{1}{2} \cos 2 \omega t+\frac{3}{8} \cos 4 \omega t\right)\right] x \\
& \left.+a_{3} x^{3}+a_{4} \dot{x}^{3}+M_{1} \cos \omega t\right\} .
\end{aligned}
$$

Attention is paid to the fundamental resonance, since the motor's angular frequency is close to the first natural frequency of the system. The frequency can be written as $\omega=$ $\omega_{0}+\varepsilon \sigma$, where $\sigma$ is the detuning parameter, which represents the proximity between $\omega$ and $\omega_{0}$.

Considering the case of the first approximation, we assume an expansion of the form

$$
x(t)=x_{0}\left(T_{0}, T_{1}\right)+\varepsilon x_{1}\left(T_{0}, T_{1}\right),
$$

where the created time scales $T_{0}=t$ and $T_{1}=\varepsilon t$.

Substituting (12) into (11) and equating the coefficients of the same power of $\varepsilon$ on both sides of the equations, we can obtain

$$
D_{0}^{2} x_{0}+\omega_{0}^{2} x_{0}=0
$$

$$
\begin{aligned}
& D_{0}^{2} x_{1}+\omega_{0}^{2} x_{1} \\
& =-2 D_{0} D_{1} x_{0}-a_{1} D_{0} x_{0} \\
& +a_{2}\left[\sin ^{2} \alpha \cos 2 \omega T_{0}\right. \\
& \quad-\sin ^{4} \alpha\left(\frac{1}{8}-\frac{1}{2} \cos 2 \omega T_{0}\right. \\
& \left.\left.\quad+\frac{3}{8} \cos 4 \omega T_{0}\right)\right] x_{0} \\
& -a_{3} x_{0}^{3}-a_{4}\left(D_{0} x_{0}\right)^{3}+M_{1} \cos (\omega t),
\end{aligned}
$$

where $D_{n}=\partial / \partial T_{n}, n=0,1, \ldots$

The general solution of (13) can be written in the form

$$
x_{0}=A\left(T_{1}\right) e^{i \omega_{0} T_{0}}+\bar{A}\left(T_{1}\right) e^{-i \omega_{0} T_{0}}
$$

where $A\left(T_{1}\right)$ and $\bar{A}\left(T_{1}\right)$ are the mutual conjugate terms; substituting (15) and $\omega=\omega_{0}+\varepsilon \sigma$ into (14), we can obtain

$$
\begin{aligned}
D_{0}^{2} x_{1}+ & \omega_{0}^{2} x_{1} \\
= & -2 i \omega_{0} D_{1} A-a_{1} i \omega_{0} A+\frac{1}{2} a_{2} \sin ^{2} \alpha \bar{A} e^{i 2 \sigma T_{0}} \\
& -\frac{1}{8} a_{2} \sin ^{4} \alpha A+\frac{1}{4} a_{2} \sin ^{4} \alpha \bar{A} e^{i 2 \sigma T_{0}} \\
& \left.-3 a_{3} A^{2} \bar{A}-3 i a_{4} \omega_{0}^{3} A^{2} \bar{A}+\frac{1}{2} M_{1} e^{i \sigma T_{0}}\right] e^{i \omega_{0} T_{0}} \\
+ & {\left[\frac{1}{2} a_{2} \sin ^{2} \alpha A e^{i 2 \sigma T_{0}}+\frac{1}{4} a_{2} \sin ^{4} \alpha A e^{i 2 \sigma T_{0}}\right.} \\
& \left.-a_{3} A^{3}+i a_{4} \omega_{0}^{3} A^{3}\right] e^{i 3 \omega_{0} T_{0}} \\
- & \frac{3}{16} a_{2} \sin ^{4} \alpha A e^{i 4 \sigma T_{0}} e^{i 5 \omega_{0} T_{0}}+\mathrm{cc},
\end{aligned}
$$

where $\mathrm{cc}$ denotes the complex conjugate of the preceding terms.

Removing the secular terms of (16), we can obtain

$$
\begin{aligned}
2 i \omega_{0} D_{1} A= & -a_{1} i \omega_{0} A+\frac{1}{2} a_{2} \sin ^{2} \alpha \bar{A} e^{i 2 \sigma T_{0}} \\
& -\frac{1}{8} a_{2} \sin ^{4} \alpha A+\frac{1}{4} a_{2} \sin ^{4} \alpha \bar{A} e^{i 2 \sigma T_{0}} \\
& -3 a_{3} A^{2} \bar{A}-3 i a_{4} \omega_{0}^{3} A^{2} \bar{A}+\frac{1}{2} M_{1} e^{i \sigma T_{0}} .
\end{aligned}
$$

Function $A$ is derived from $\left(d A / d T_{1}\right)=\left(D_{0}+\varepsilon D_{1}\right) A$, where $D_{0} A=0$, so $D_{1} A$ can be obtained by (17); then

$$
\begin{aligned}
\frac{d A}{d T_{1}}=\frac{-i}{2}\{ & -a_{1} i A+\left(\frac{1}{2} \frac{a_{2}}{\omega_{0}} \sin ^{2} \alpha+\frac{1}{4} \frac{a_{2}}{\omega_{0}} \sin ^{4} \alpha\right) \\
& \cdot \bar{A} e^{i 2 \sigma T_{0}} \frac{1}{8} \frac{a_{2}}{\omega_{0}} \sin ^{4} \alpha A-\frac{3 a_{3} A^{2} \bar{A}}{\omega_{0}} \\
& \left.-3 i a_{4} \omega_{0}^{2} A^{2} \bar{A}+\frac{1}{2 \omega_{0}} M_{1} e^{i \sigma T_{0}}\right\} .
\end{aligned}
$$


Letting $A=(1 / 2) a e^{i \theta}$ in (18), where $a$ and $\theta$ are real functions, and $\gamma=\sigma T_{0}-\theta$. Separating real parts and imaginary parts, we get

$$
\begin{aligned}
\dot{a}= & -\frac{1}{2} a_{1} a+\left(\frac{1}{4} \frac{a_{2}}{\omega_{0}} a \sin ^{2} \alpha+\frac{1}{8} \frac{a_{2}}{\omega_{0}} a \sin ^{4} \alpha\right) \sin 2 \gamma \\
& -\frac{3}{2} a_{4} \omega_{0}^{2} a^{3}+\frac{1}{4 \omega_{0}} M_{1} \sin \gamma, \\
a \dot{\gamma}= & \sigma a+\left(\frac{1}{4} \frac{a_{2}}{\omega_{0}} a \sin ^{2} \alpha+\frac{1}{8} \frac{a_{2}}{\omega_{0}} a \sin ^{4} \alpha\right) \cos 2 \gamma \\
& -\frac{3}{8 \omega_{0}} a^{3} a_{3}-\frac{1}{16} \frac{a_{2}}{\omega_{0}} a \sin ^{4} \alpha+\frac{1}{4 \omega_{0}} M_{1} \cos \gamma .
\end{aligned}
$$

Steady state motions occur at $\dot{a}=\dot{\gamma}=0$, so (19) can be given as

$$
\begin{aligned}
& \left(\frac{1}{4} \frac{a_{2}}{\omega_{0}} \sin ^{2} \alpha+\frac{1}{8} \frac{a_{2}}{\omega_{0}} \sin ^{4} \alpha\right) a \sin 2 \gamma+\frac{1}{4 \omega_{0}} M_{1} \sin \gamma \\
& =\frac{1}{2} a_{1} a+\frac{3}{2} a_{4} \omega_{0}^{2} a^{3}, \\
& \left(\frac{1}{4} \frac{a_{2}}{\omega_{0}} \sin ^{2} \alpha+\frac{1}{8} \frac{a_{2}}{\omega_{0}} \sin ^{4} \alpha\right) a \cos 2 \gamma+\frac{1}{4 \omega_{0}} M_{1} \cos \gamma \\
& =-a \sigma+\frac{3}{8 \omega_{0}} a^{3} a_{3}+\frac{1}{16} \frac{a_{2}}{\omega_{0}} a \sin ^{4} \alpha .
\end{aligned}
$$

The $\gamma$ is eliminated by (20), the amplitude-frequency response equation can be given as

$$
\begin{aligned}
& \frac{8 N}{M_{1}^{2}}\left(P^{2} a^{2}+Q^{2} a^{2}-N^{2} a^{2}-\frac{1}{16} \frac{M_{1}^{2}}{\omega_{0}^{2}}\right)^{2} \\
& \quad+\frac{1}{2}\left(P^{2} a+Q^{2} a-N^{2} a-\frac{1}{16 a} \frac{M_{1}^{2}}{\omega_{0}^{2}}\right)-N-Q=0
\end{aligned}
$$

where $N=\left(a_{2} / 4 \omega_{0}\right) \sin ^{2} \alpha+\left(a_{2} / 8 \omega_{0}\right) \sin ^{4} \alpha, P=(1 / 2) a_{1}+$ $(3 / 2) a_{4} \omega_{0}^{2} a^{2}$, and $Q=-\sigma+\left(3 / 8 \omega_{0}\right) a^{2} a_{3}+(1 / 16)\left(a_{2} / \omega_{0}\right) \sin ^{4} \alpha$.

\section{Numerical Example and Discussion}

The numerical simulation based on the main drive system of stand F7 of 1780 rolling mill of some Steel Co. simplifies the practical model into a 4-DOF torsional system. Here are the parameters of the main drive system: $J_{1}=$ $8080.081 \mathrm{~kg} \cdot \mathrm{m}^{2}, J_{2}=324.7148 \mathrm{~kg} \cdot \mathrm{m}^{2}, J_{3}=266.1488 \mathrm{~kg} \cdot \mathrm{m}^{2}$, $J_{4}=4885.4 \mathrm{~kg} \cdot \mathrm{m}^{2}, K_{1}=1.2632 \times 10^{8} \mathrm{Nm} / \mathrm{rad}, K_{1}=1.0884 \times$ $10^{8} \mathrm{Nm} / \mathrm{rad}, K_{1}=2.786 \times 10^{8} \mathrm{Nm} / \mathrm{rad}$, and $\zeta_{i}=0.02$.

Figure 2 shows the relationship between amplitude and frequency responses of the different joint angles which are $3^{\circ}, 5^{\circ}$, and $8^{\circ}$, respectively. $\sigma$ is the detuning parameter. It can be seen that there is a slight reduction of resonance peak amplitudes which reflects the joint angles decrease. A similar phenomenon is observed for the resonance curves by shifting the backbone curve to the right side with the frequency increase. These facts reveal the vibration response,

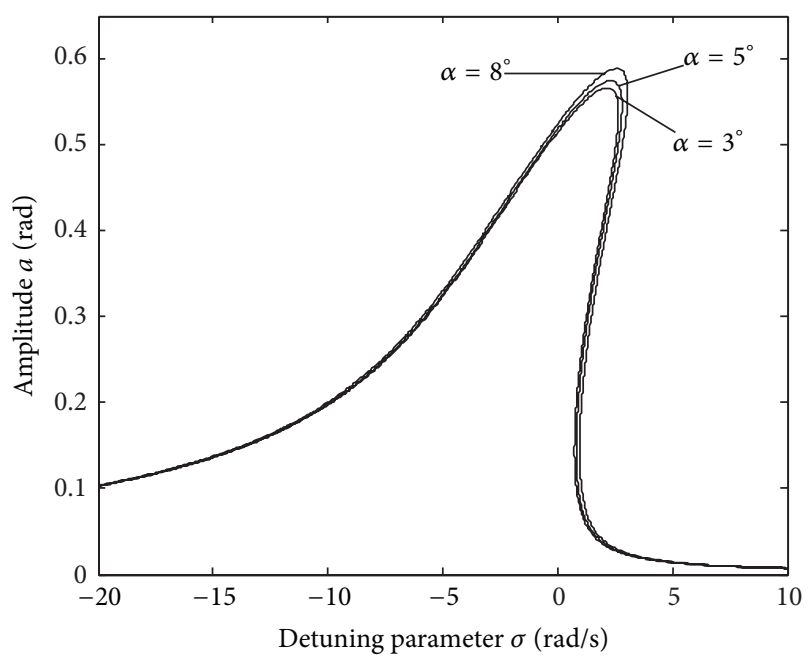

FIgURE 2: Amplitude-frequency characteristic curve.

which relates to the change of the joint angle of the main drive system and affects the behavior of resonances.

A more comprehensive understanding on the effect of $M$ could be achieved by constructing the nonlinear frequency response characteristics. Dynamic responses may be quantified by applying the values from the calculation at each excitation frequency. Figure 3 shows the amplitudefrequency response curves in values in which $M$ is $4.1635 \times$ $10^{5} \mathrm{Nm}, 8.3269 \times 10^{5} \mathrm{Nm}$, and $1.1776 \times 10^{6} \mathrm{Nm}$ and the joint angle is $5^{\circ}$. Since there is no spring in parallel with the external excitation, both the displacement and resonance area may grow up to a larger value under the influence of the increase of the disturbance torque.

The effect of the nonlinear damping is clearly visible in Figure 4. As $C_{\mathrm{NL}}$ decreases, the resonance zone amplifies and the peak amplitude increases. The backbone curves do not bend or fold. A comparison of the resonance curves with different nonlinear stiffness in Figure 5 shows diverse changes. Unlike the case in Figure 4 where the peak amplitude increases, the amplitudes almost keep a constant but the backbone curves are evidently bended to the right side with the value of $K_{\mathrm{NL}}$ aggrandized. Both the nonlinear damping and nonlinear stiffness play active roles in the resonance regime, respectively.

Figure 6 shows the relationship between joint angles and disturbance torque. Such a bended trend of the curve is consistent with the amplitude gradually growing. Higher amplitude of the resonance regime can be explained by the fact that the larger joint angle under the same disturbance receives more excitation.

When only interested in the relationship between amplitude and the value of $\sin \alpha$, it cannot be obtained unless the value of the detuning parameter $\sigma$ is known. Comparisons disturbance torque, nonlinear damping, and nonlinear stiffness are clearly shown in Figures 7, 8, and 9, respectively. All the values of the detuning parameter here are defined to be 1 .

As discussed before, the amplitude goes up with the disturbance torque increasing under the lower value regime 


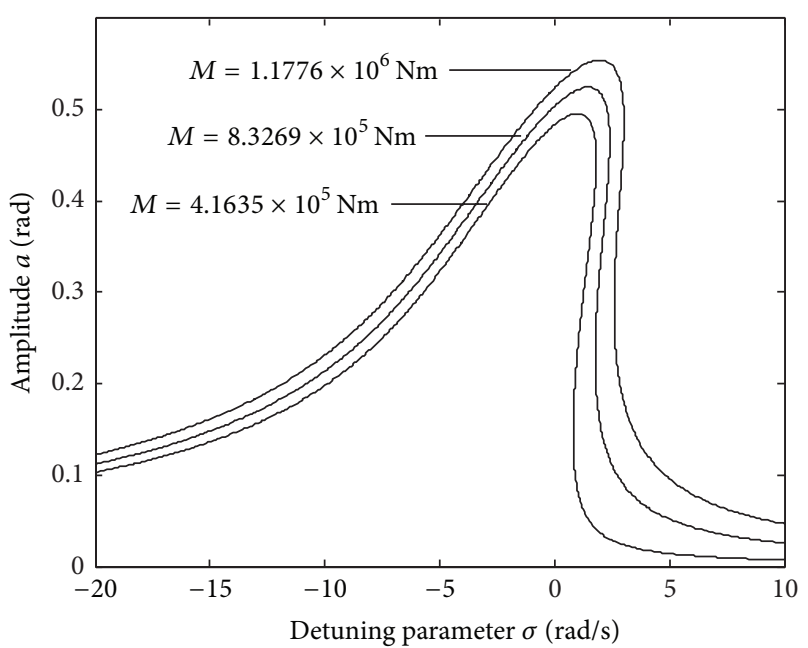

FIgURE 3: Amplitude-frequency characteristic curve.

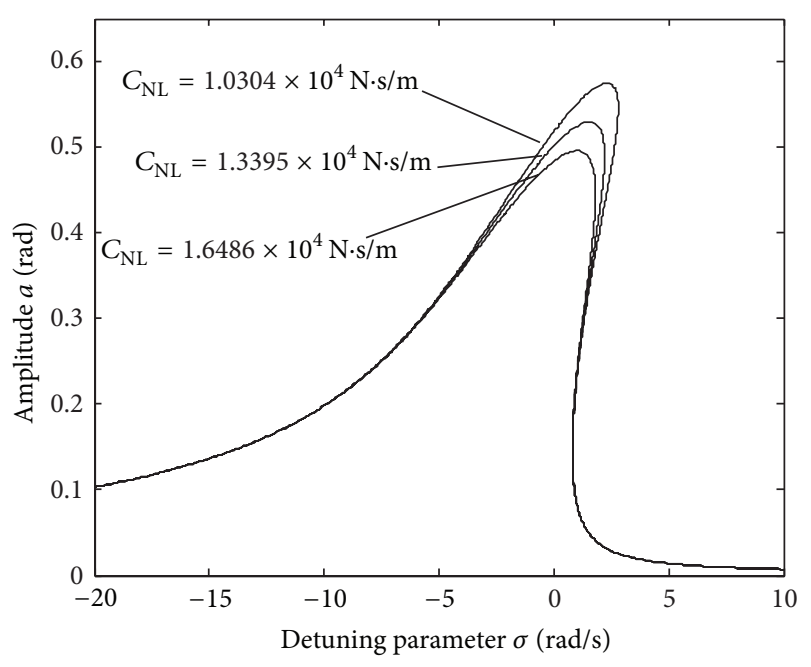

FIgURE 4: Amplitude-frequency characteristic curve.

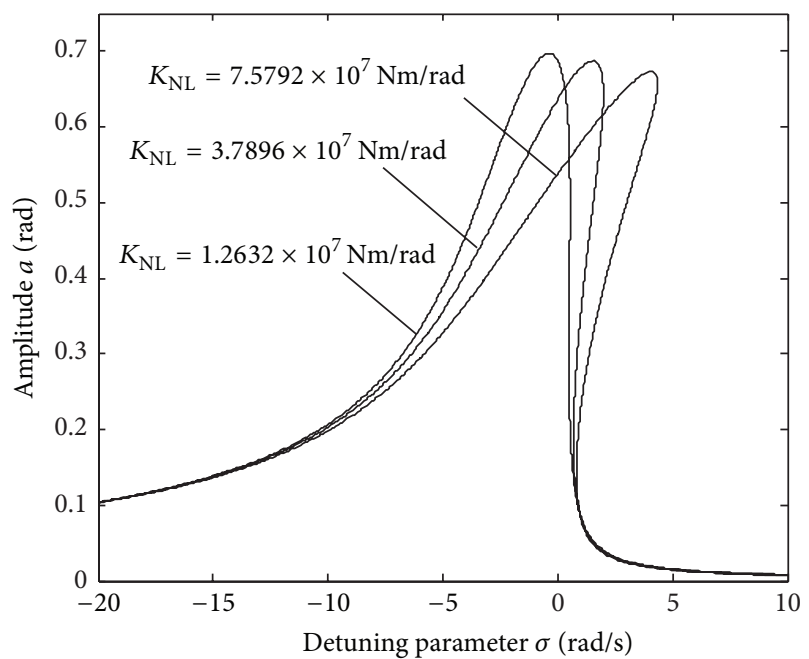

FIGURE 5: Amplitude-frequency characteristic curve.

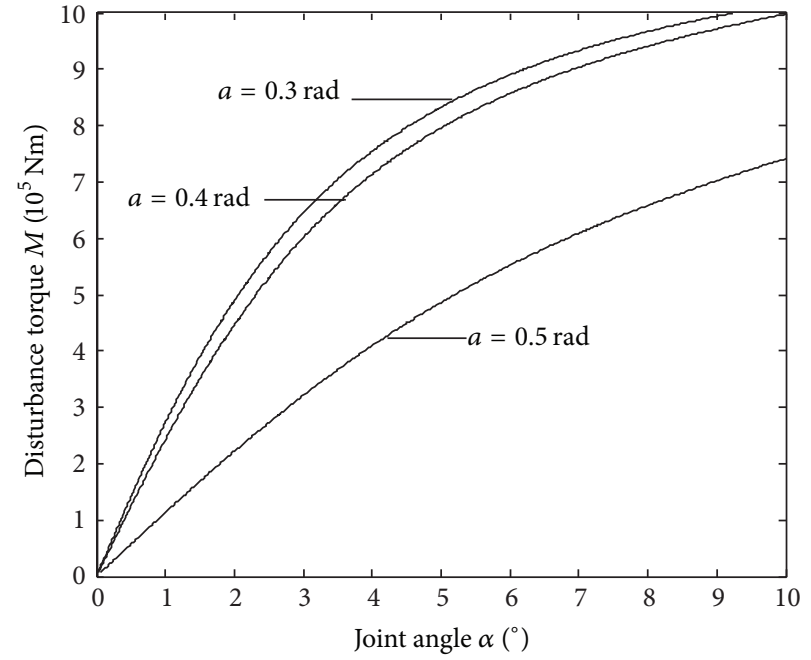

FIGURE 6: Disturbance torque-angle characteristic curve.

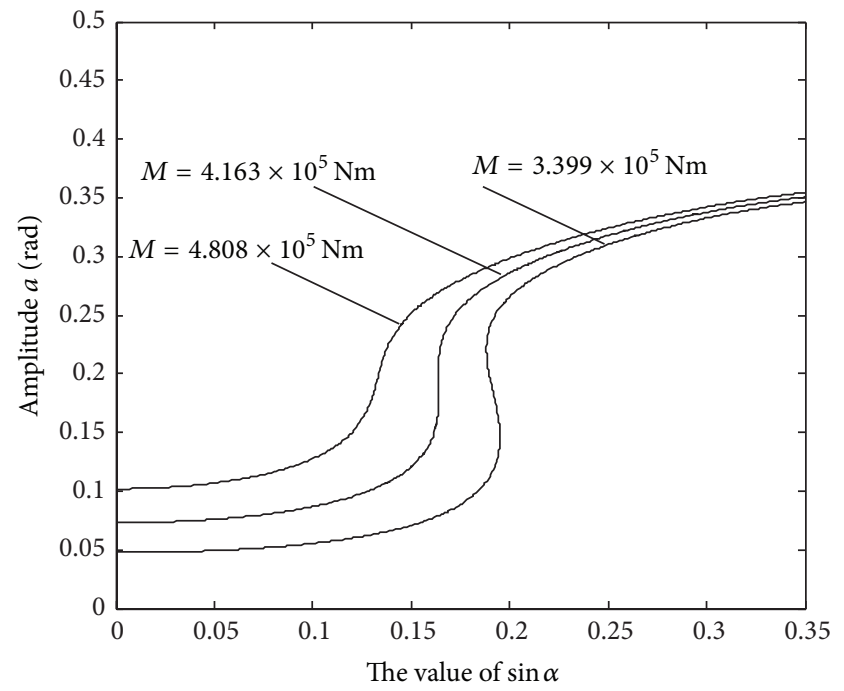

Figure 7: Amplitude-angle characteristic curve.

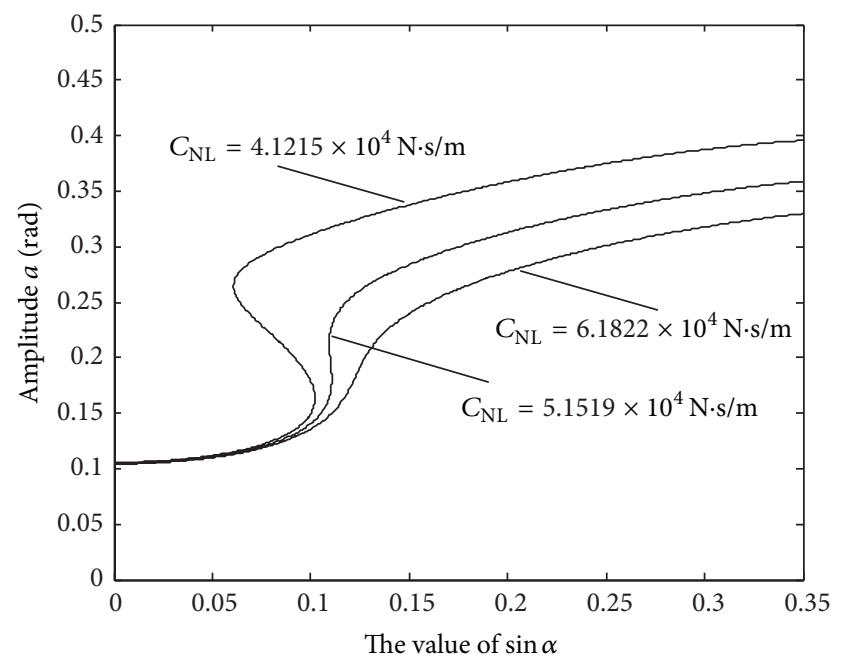

Figure 8: Amplitude-angle characteristic curve. 


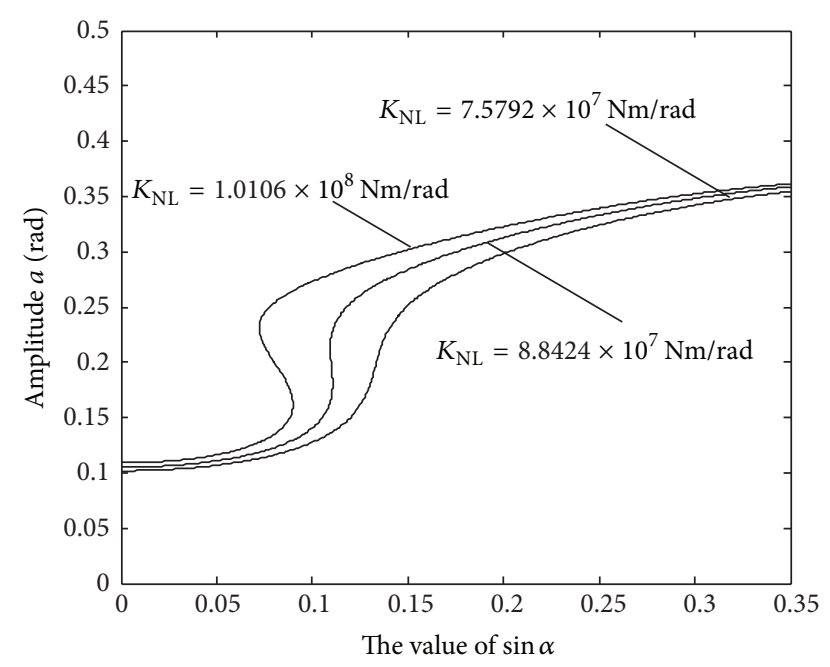

FIgURE 9: Amplitude-angle characteristic curve.

of $\sin \alpha$. In Figure 7 sample results are shown. The amplitude increases gradually when the value of $\sin \alpha$ enlarges to 0.15 , which is named critical value here, and it runs up to a higher value rapidly when the value of $\sin \alpha$ exceeds 0.13 under $M=3.399 \times 10^{5} \mathrm{Nm}$. Note that this is higher than the one previously discussed when $M=4.163 \times 10^{5} \mathrm{Nm}$. The similar response pattern, a declining effect followed, is observed. Unlike the influence under the slighter disturbance torque condition, the critical value becomes smaller. The critical value is nearly 0.12 under $M=4.808 \times 10^{5} \mathrm{Nm}$. The simulated value of the joint angle is at most $8^{\circ}$, under which the system could vibrate at relative lower amplitude.

The same phenomenon can be seen in Figures 8 and 9 as it performs in Figure 7. But each condition has a different critical value in different amplitude-angle characteristic curve. The same jump phenomenon is expected in the larger value of $\sin \alpha$ regime that exhibits instability. The curves show a drastic jump from the critical value due to a finite area in the figures.

In Figure 8, the amplitudes are almost equal at a lower value of $\sin \alpha$ under different nonlinear damping. With the increase of the value of $\sin \alpha$, the relative smaller nonlinear damping has the lower critical value. The critical value in the vicinity is quite unstable as shown in Figure 8 . The variation is a little dissimilar to the performance in Figure 9. The amplitudes are distinct at a lower value of $\sin \alpha$ under different nonlinear stiffness. The smaller critical value is employed by the larger stiffness.

\section{Conclusions}

In this paper, the nonlinear torsional vibration dynamical modeling of the multi-DOF rolling mill's main drive system under joint angle parametric excitation is established. The influence of joint angle in the rolling mill's main drive system is taken into account and expressed by parametric stiffness. Attention is paid to the fundamental resonance, since the excitation frequency is close to the first natural frequency of the system.
The multiscale method is used to evaluate the nonlinear dynamic behavior of the torsional system by obtaining the amplitude-frequency characteristic equation and amplitudefrequency response equation. Numerical example of the 1780 rolling mill of some Steel Co. rolling mill's main drive system is given to illustrate the effects of the resonance on the response of the system. The effects of the joint angle, nonlinear damping, nonlinear stiffness, and the disturbance torque on the amplitude of the system are discussed. The limited joint angles range in the rolling process is obtained, and the variation rules of the joint angle caused by the nonlinear damping, nonlinear stiffness, and the disturbance torque are given.

The results presented the rolling mill can work more stably with the joint angle at a range from $2^{\circ}$ to $8^{\circ}$ by controlling the value of parameters. Overall, our analysis matches the practical parameters in a qualitative manner.

\section{Conflict of Interests}

The authors declare that there is no conflict of interests regarding the publication of this paper.

\section{Acknowledgments}

This work was supported by the National Natural Science Foundation of China under Grants 51104129 and 51005196 and Hebei Provincial Natural Science Foundation of China (no. E2012203194).

\section{References}

[1] L. Ng and R. Rand, "Nonlinear effects on coexistence phenomenon in parametric excitation," Nonlinear Dynamics, vol. 31, no. 1, pp. 73-89, 2003.

[2] Z. Gao, D. Kong, and C. Gao, "Modeling and control of complex dynamic systems: applied mathematical aspects," Journal of Applied Mathematics, vol. 2012, Article ID 869792, 5 pages, 2012.

[3] Z. Gao, D. Kong, C. Gao, and M. Chen, "Modeling and control of complex dynamic systems," Journal of Applied Mathematics, vol. 2013, Article ID 151372, 4 pages, 2013.

[4] A. A. Jafari, A. K. Tieu, and A. Basu, "Asymmetrical rolling and self excited vibrations in a hot roughing mill," American Society of Mechanical Engineers, vol. 60, no. 7, pp. 193-202, 1993.

[5] Z. Wang and D. Wang, "Method of judging the self-excited vibration of rolling main drive system in rolling slippage," Journal of Sound and Vibration, vol. 215, no. 5, pp. 1135-1143, 1998.

[6] Z.H. Wang and D. J. Wang, "Dynamic characteristics of a rolling mill drive system with backlash in rolling slippage," Journal of Materials Processing Technology, vol. 97, no. 1-3, pp. 69-73, 2000.

[7] H. P. Tang, D. Y. Wang, and J. Zhong, "Investigation into the electromechanical coupling unstability of a rolling mill," Journal of Materials Processing Technology, vol. 129, no. 1-3, pp. 294-298, 2002.

[8] Z. Drzymala, A. Świaţoniowski, and A. Bar, "Non-linear vibrations in cold rolling mills," Mécanique et Industries, vol. 4, no. 2, pp. 151-158, 2003.

[9] R. Guo, S. Jang, and Y. Choi, "Torsional vibration analysis of lathe spindle system with unbalanced workpiece," Journal of 
Central South University of Technology, vol. 18, no. 1, pp. 171-176, 2011.

[10] T. Tamaoki, M. Takanezawa, M. Kimoto, N. Morita, T. Hoshino, and K. Hashizume, "Study on online analysis of transfer function of variable-speed rolling mill motor with shaft torsional vibration systems," IEEJ Transactions on Industry Applications, vol. 131, no. 6, pp. 844-857, 2011.

[11] P. Krot, "Nonlinear vibrations and backlashes diagnostics in the rolling mills drive trains," in Proceedings of the 6th EUROMECH Nonlinear Dynamics Conference (ENOC '08), pp. 26-30, Saint Petersburg, Russia, 2008.

[12] H. George, Kinematics and Dynamics of Machines, McGrawHill, New York, NY, USA, 1969. 


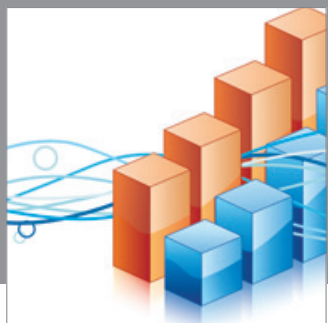

Advances in

Operations Research

mansans

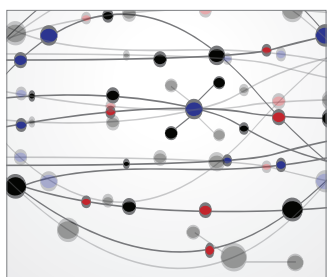

The Scientific World Journal
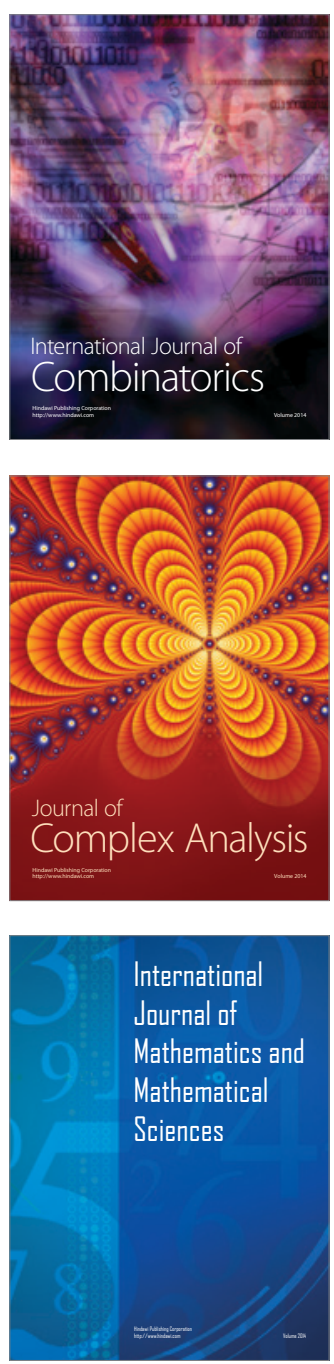
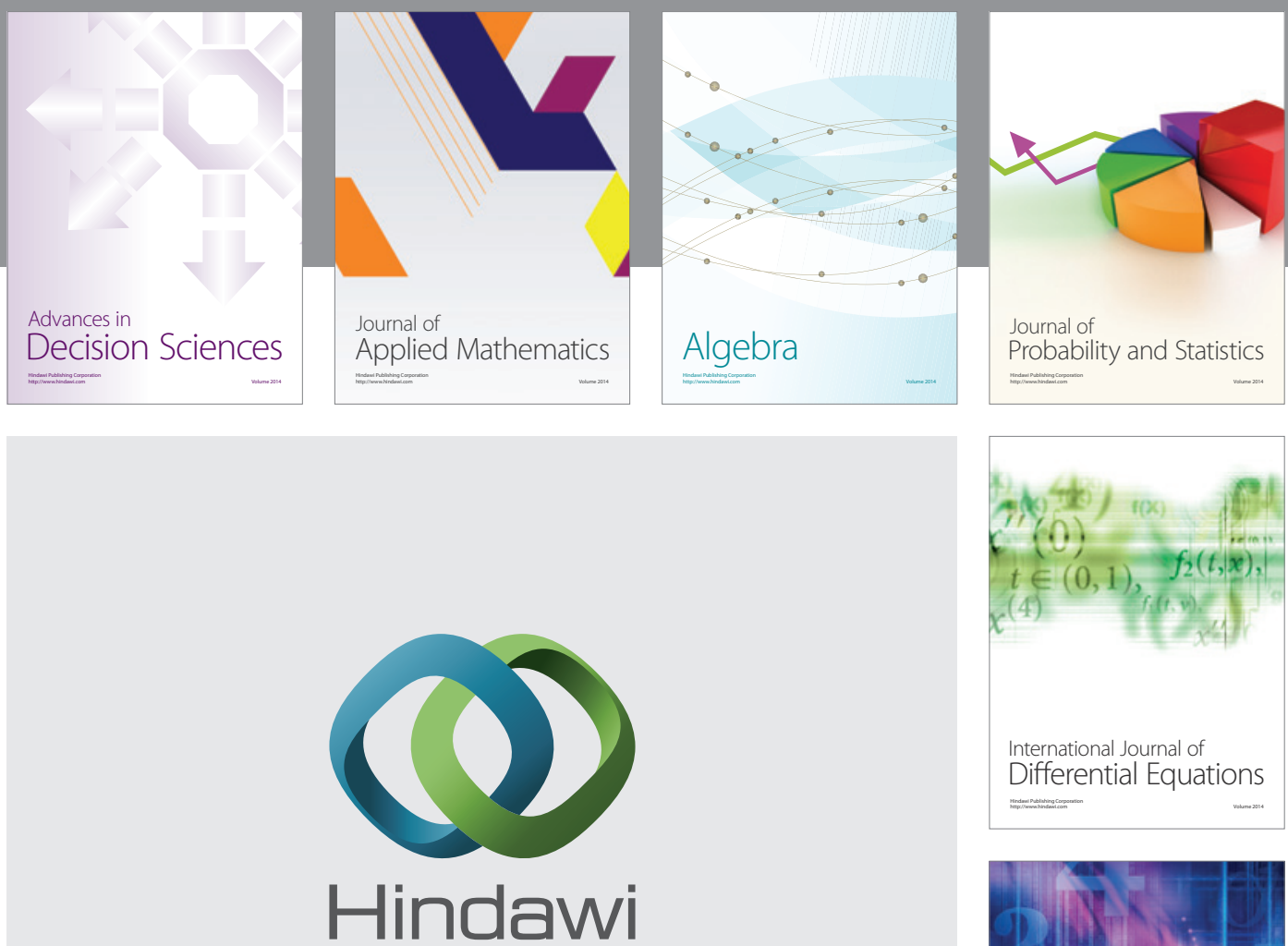

Submit your manuscripts at http://www.hindawi.com
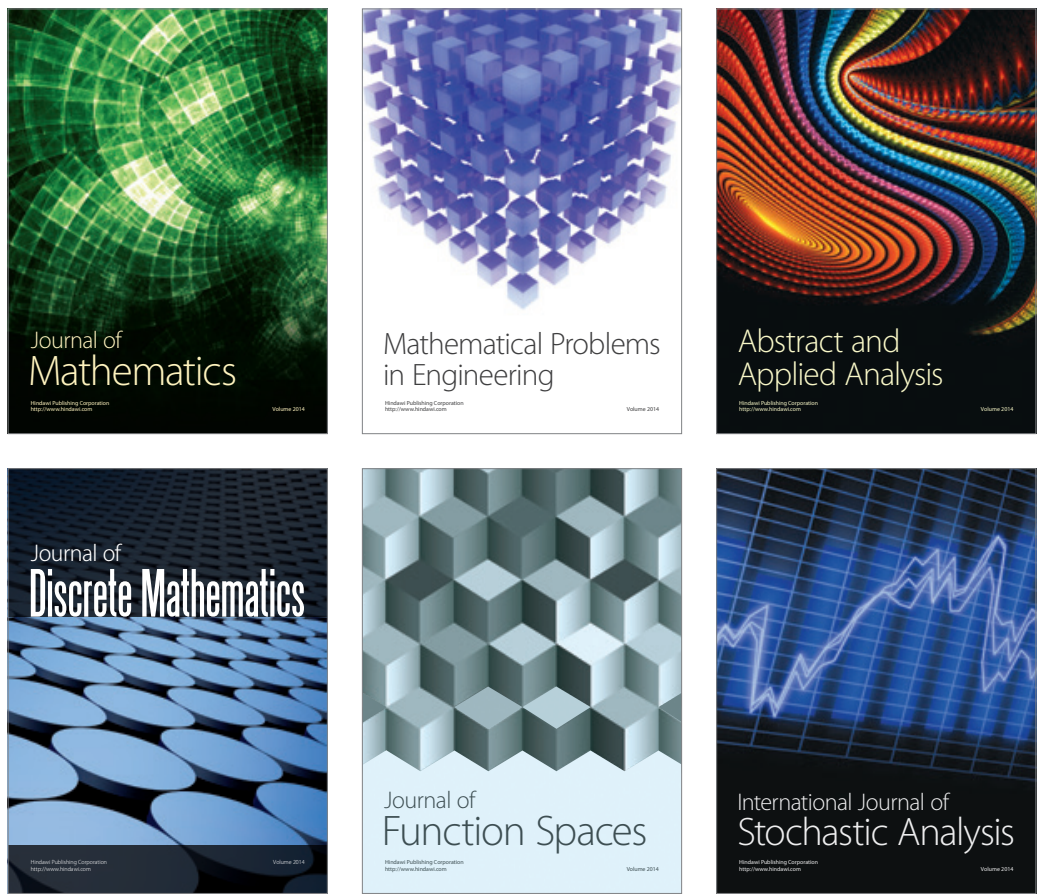

Journal of

Function Spaces

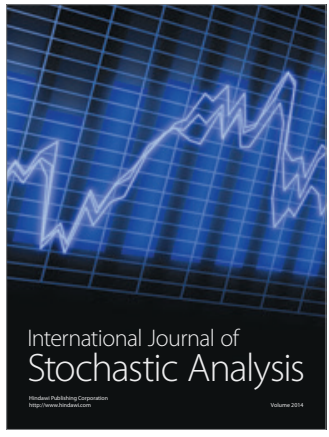

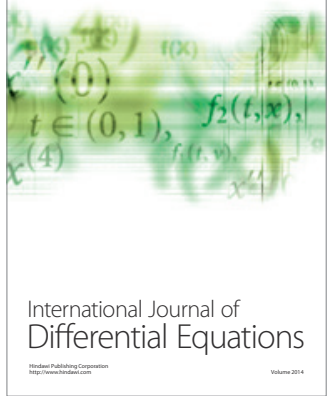
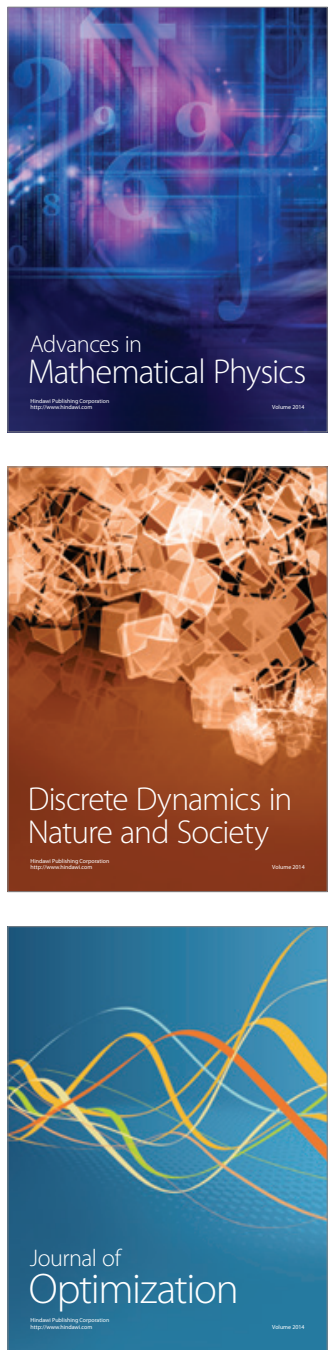